\title{
Arteriovenous Fistula as a Rare Early Complication of Transradial Coronary Angiography: With Literature Review on Management Strategies
}

\author{
David Chieng $^{\mathrm{a}, \mathrm{b}}$, Bhadresh Patel ${ }^{\mathrm{a}}$
}

\begin{abstract}
Iatrogenic arteriovenous (AV) fistula is a vascular complication which may result from coronary angiography. Whilst infrequent via transfemoral access, the incidence in transradial angiography is even rarer with mostly case reports in the literature. Most of these had thus far described transradial AV fistula formation as a delayed complication requiring surgical intervention. Here we describe a rare early case of transradial AV fistula complicating coronary intervention which was managed conservatively with mechanical compression. Follow-up imaging confirmed resolution of fistula. We also reviewed the existing literature on management strategies for transradial AV fistula. We support the use of conservative management as the firstline treatment option for transradial AV fistula, with surgical options reserved for cases involving limb vascular compromise and cardiac insufficiency.
\end{abstract}

Keywords: Transradial; Angiography; Arteriovenous fistula; Conservative; Ligation

\section{Introduction}

Transradial access for coronary angiography and intervention has become increasingly popular and is even recommended as the default coronary intervention strategy by the European Association of Percutaneous Cardiovascular Interventions (EAPCI) [1]. Access site vascular complications, including hematomas, retroperitoneal hematomas, arteriovenous (AV) fistula, pseudoaneurysms, and arterial dissection/occlusion have all been shown to be significantly reduced via the transradial approach compared to the transfemoral approach [1-4].

$\mathrm{AV}$ fistula formation is caused by needle deviation through

Manuscript accepted for publication September 08, 2015

${ }^{a}$ Western Cardiology, St John of God Subiaco Hospital, Perth, Western Australia

${ }^{\mathrm{b}}$ Corresponding Author: David Chieng, Western Cardiology, St John of God Hospital, Perth, Western Australia. Email: davidchieng87@gmail.com

doi: http://dx.doi.org/10.14740/jmc2291w a vein resulting in a combined artery and vein puncture [2]. If this communication fails to heal, then a fistula is formed. The majority of literature on transradial AV fistula is based on case reports, with timing of AV fistula diagnosis ranging from weeks up to 1 year post-angiography. We present a rare case of AV fistula formation as an early complication of transradial angiography.

\section{Case Report}

A 76-year-old man with a background history of mechanical aortic valve replacement (AVR) on warfarin anticoagulation presented with syncopal episodes and chest pains. He had a permanent pacemaker (PPM) inserted for sinus bradycardia with trifascicular block on ECG. He also underwent diagnostic transradial coronary angiography using a 5-French radial sheath with Judkins 3.5 catheters (Terumo Medical Corporation) which revealed significant calcification and stenosis in mid right coronary artery (RCA). A decision was made for elective rotablation and stenting of RCA 1 month post-PPM with pre-procedural warfarin cessation and bridging anticoagulation via clexane. No complication was noted following initial angiography.

The rotablation and stenting to the RCA was again performed via right radial approach using 6-French radial sheath (Terumo Medical Corporation). Pre-procedural Allen's test was normal. Anticoagulation was administered with heparin 12,000 units. The RCA lesion was wired with a 0.014 inch Runthrough Extra Floppy wire (Terumo Medical Corporation) with balloon exchange to a Rota-Floppy wire (Boston Scientific). The heavily calcified RCA lesion was rotablated with a $1.5 \mathrm{~mm}$ burr (Boston Scientific), following which pre-dilation was performed with serial noncompliant balloons (Boston Scientific; Abbott Vascular). A $3.5 \times 28 \mathrm{~mm}$ bare metal multi-link stent (Abbott Vascular) was deployed and post-dilation was done with a $3.5 \mathrm{~mm}$ balloon to 18 atmospheres pressure (Abbott Vascular). The radial artery sheath was removed immediately after the procedure and hemostasis was achieved by application of an adjustable plastic clamp of the radial artery (TR Terumo band). The clamp was gradually released over few hours while the access site was monitored for bleeding and hematoma. Patient was commenced on dual antiplatelet therapy (aspirin and clopidogrel), and his warfarin was recom- 


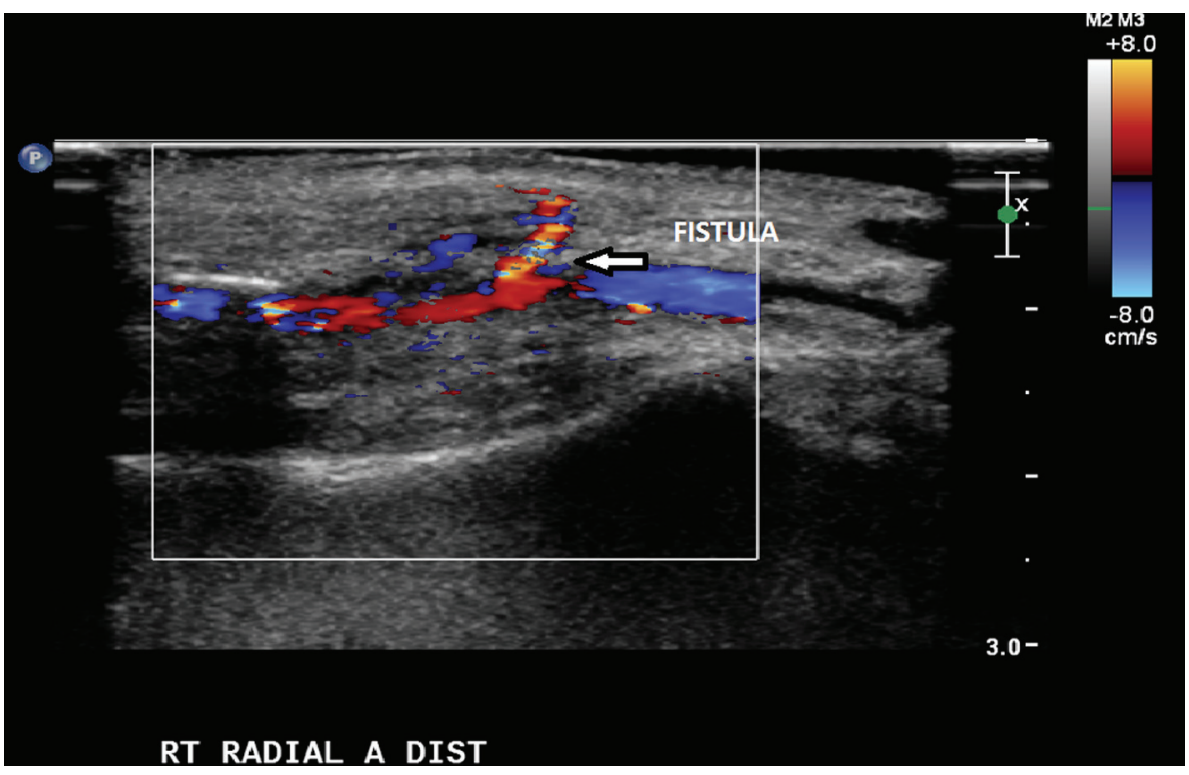

Figure 1. Doppler ultrasound of right radial artery (sagittal view) showing AV fistula connecting the radial artery to the distal cephalic vein (arrow).

menced with bridging clexane cover.

On day 5 post-procedure, patient complained of pulsatile swelling with minimal tenderness on palpation over the right radial artery. He did not complain of finger claudication or numbness, and the Allen's test remained normal. An ultrasound Doppler was arranged which confirmed small AV fistula connecting the right radial artery with a superficial vein over the access site (Fig. 1). The AV fistula was managed conservatively with TR Terumo band application for $24 \mathrm{~h}$ and limb elevation. No alteration to his anti-thrombotic therapy was made. The patient had a follow-up Doppler US after 1 month which showed resolution of the AV fistula (Fig. 2).

\section{Discussion}

The benefits of using radial artery as angiography access, which is a superficial and readily compressible artery compared to the femoral artery [2], have been shown in multiple observational and randomized controlled studies. The 2012 randomized controlled RIVAL study showed that access site complications were significantly less in the transradial group

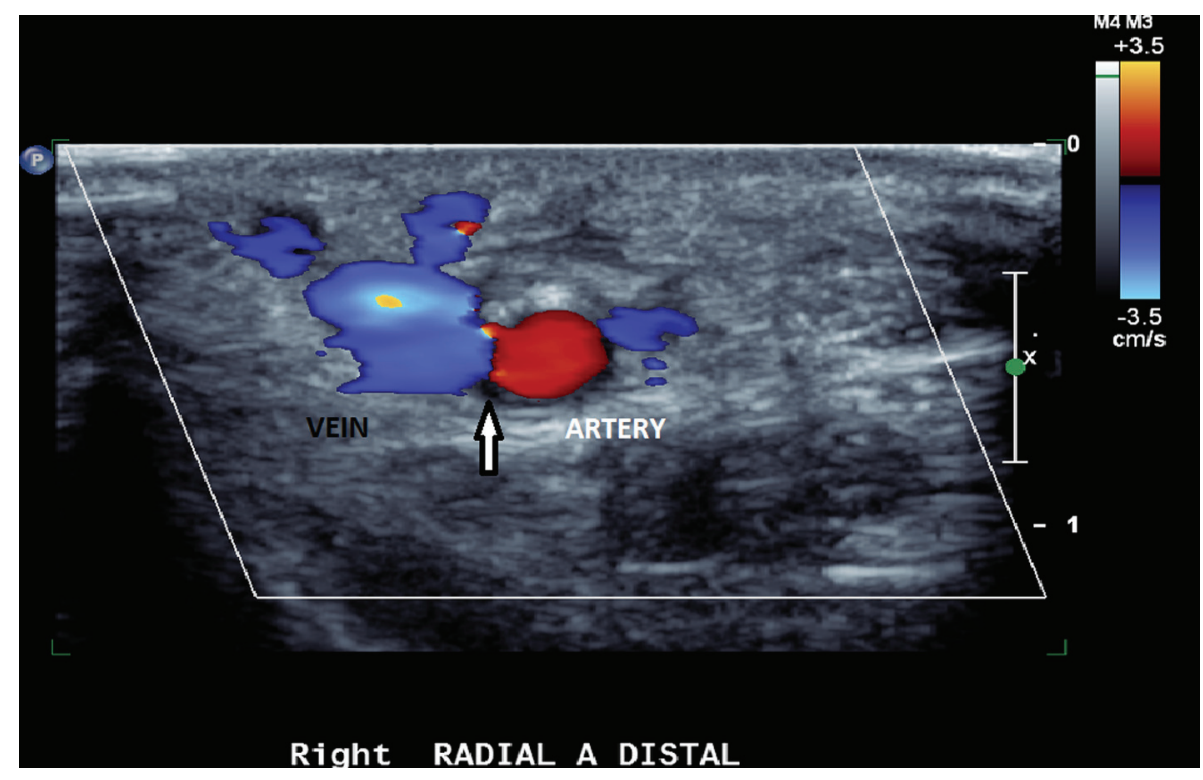

Figure 2. Repeat Doppler ultrasound of right radial artery (transverse view) showing resolution of AV fistula with no flow between radial artery and cephalic vein (arrow). 
Table 1. Reported Cases of Transradial Arteriovenous Fistula and Management Strategy

\begin{tabular}{llll}
\hline Study & Patient & AV fistula diagnosis (from time of angiography) & Treatment \\
\hline Tatli et al [1] & 78-year-old female & 3 months & Surgical ligation \\
Yang et al [4] & 54-year-old male & 2 months & Surgical ligation \\
Spence et al [6] & 61-year-old male & 1 year & Surgical ligation \\
Na et al [8] & 61-year-old female & 1 year & Surgical ligation \\
Kwac et al [9] & 67-year-old male & 1 year & Surgical ligation \\
Dehghani et al [2] & 62-year-old male & 1 month & Conservative \\
Tatli et al [1] & 71-year-old female & 3 weeks & Conservative (compression) \\
& 64-year-old male & 2 weeks & Conservative (compression) \\
& 55-year-old female & 1 month & Conservative (compression) \\
\hline
\end{tabular}

(1.4\%) compared to the transfemoral group (3.8\%) [5]. Similar rates have been reported by a systematic review with vascular complication rates of $0.59 \%$ via transradial approach compared to $3.71 \%$ via transfemoral approach [3]. In addition, transradial access is associated with earlier ambulation, greater patient comfort, and earlier discharges from the hospital $[2,3]$.

The low incidence of AV fistula formation complicating transradial coronary angiography is illustrated in a recent large case series of 10,324 patients by Tatli et al, where only four cases of AV fistula were documented [1]. Patients with iatrogenic radial AV fistula are usually asymptomatic and typically present with a pulsatile mass, thrill, or bruit over the wrist $[6$, 7]. The time to diagnosis can be variable from weeks up to a year from the angiography procedure. In our case, our patient first noticed his pulsatile mass 5 days after his procedure, which was relatively early in onset.

Duplex ultrasonography is the routine screening test for patients with suspected AV fistula [8]. Factors have been identified which increases the risk of iatrogenic femoral AV fistula, although it is unclear whether they apply to transradial angiography as well. These factors were female sex, age more than 60, hypertension, prolonged warfarin therapy, and high heparin dose during interventions [2]. In line with this, recommendations have been quoted in the literature to potentially reduce the risk of iatrogenic AV fistula formation, including use of sheath sizes less than the arterial diameter, limitation on the number of arterial puncture attempts, gentle catheter manipulation and use of ultrasound guided needle placement to provide real time anatomic information on proximity of the radial artery to nearby veins $[2,3]$.

This report also reviewed existing literature on management strategies for iatrogenic radial AV fistula. The natural history of radial $\mathrm{AV}$ fistula is thought to be benign with most cases resolving spontaneously $[1,2]$, although serious consequences in the form of high output cardiac failure and distal limb ischemia can potentially occur. As in transfemoral AV fistula, three management strategies have been explored in transradial AV fistula, namely conservative management (including mechanical compression), implantation of covered stents and surgical repair [2].

Earlier case reports on radial AV fistula have all utilized the use of surgical ligation, with a range of presentation from 2 months post-angiography up to 1 year $[1,2,4,6,8,9]$ (Table
1). Out of the four AV fistula cases from Tatli et al, only one case required surgical ligation [1]. No complications from the surgical ligation itself were identified from these cases. The authors of this report have identified one case involving the use a covered stent [10]. However, unlike transfemoral cases, the practicality of this technique is limited by the small size of the radial artery, and the potential risk of restenosis [2].

Conservative management of radial AV fistula was reported on a patient who had this complication 1 month post-angiography [2]. The fistula failed to resolve on 1-year follow-up but due to ongoing stable hemodynamic status, the management approach was continued. Seventy-five percent of the radial AV fistula cases reported by Tatli et al resolved with mechanical compression for $72 \mathrm{~h}$ and vertical arm rest [1]. In view of this, these studies have recommended that a conservative approach be the first-line treatment option for patients with AV fistula formation. Our experience with this early case of AV fistula provides further support to this management approach, with successful resolution following conservative treatment mechanical compression for $24 \mathrm{~h}$.

In conclusion, AV fistula is a rare complication from transradial angiography. The authors of this report agree that a conservative approach should be the default first-line treatment option for patient with this unusual complication. Invasive approaches, in turn, should be reserved for patients who have evidence of limb ischemia and/or cardiac insufficiency.

\section{Conflict of Interest}

The authors have no conflict of interest to declare.

\section{References}

1. Tatli E, Buturak A, Cakar A, Vatan BM, Degirmencioglu A, Agac TM, Kilic H, et al. Unusual Vascular Complications Associated with Transradial Coronary Procedures Among 10,324 Patients: Case Based Experience and Treatment Options. J Interv Cardiol. 2015;28(3):305-312.

2. Dehghani P, Culig J, Patel D, Kraushaar G, Schulte P. Arteriovenous fistula as a complication of transradial coronary angiography: a case report. J Med Case Rep. 
2013;7:21.

3. Agostoni P, Biondi-Zoccai GG, de Benedictis ML, Rigattieri S, Turri M, Anselmi M, Vassanelli C, et al. Radial versus femoral approach for percutaneous coronary diagnostic and interventional procedures; Systematic overview and meta-analysis of randomized trials. J Am Coll Cardiol. 2004;44(2):349-356.

4. Yang JH, Gwon HC, Park JE, Song YB. Arteriovenous fistula of the wrist after transradial coronary intervention. Heart Lung. 2012;41(2):203-206.

5. Jolly SS, Yusuf S, Cairns J, Niemela K, Xavier D, Widimsky P, Budaj A, et al. Radial versus femoral access for coronary angiography and intervention in patients with acute coronary syndromes (RIVAL): a randomised, parallel group, multicentre trial. Lancet. 2011;377(9775):14091420.

6. Spence MS, Byrne J, Haegeli L, Mildenberger R, Kinloch
D. Rare access site complications following transradial coronary intervention. Can J Cardiol. 2009;25(6):e206.

7. Kanei Y, Kwan T, Nakra NC, Liou M, Huang Y, Vales LL, Fox JT, et al. Transradial cardiac catheterization: a review of access site complications. Catheter Cardiovasc Interv. 2011;78(6):840-846.

8. Na KJ, Kim MA, Moon HJ, Lee JS, Choi JS. Radial arteriovenous fistula developed late after coronary angiography: a case report. Korean J Thorac Cardiovasc Surg. 2012;45(6):421-423.

9. Kwac MS, Yoon SJ, Oh SJ, Jeon DW, Kim DH, Yang JY. A rare case of radial arteriovenous fistula after coronary angiography. Korean Circ J. 2010;40(12):677-679.

10. Summaria F, Romagnoli E, Preziosi P. Percutaneous antegrade transarterial treatment of iatrogenic radial arteriovenous fistula. J Cardiovasc Med (Hagerstown). 2012;13(1):50-52. 